\title{
Analogues of Rayleigh-Taylor and Richtmyer-Meshkov instabilities in flows with nonuniform particle and droplet seeding
}

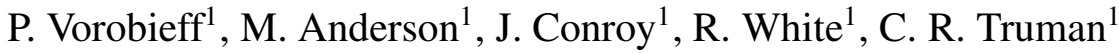 \\ \& S. Kumar ${ }^{2}$ \\ ${ }^{I}$ Department of Mechanical Engineering, The University of New Mexico, \\ Albuquerque, USA \\ ${ }^{2}$ Department of Engineering, The University of Texas, Brownsville, USA
}

\begin{abstract}
The well-known Rayleigh-Taylor (RT) and Richtmyer-Meshkov (RM) instabilities characterize the behavior of flows where two gases (or fluids) of different densities mix due to gravity (RT) or due to impulsive acceleration (RM). Recently, analogous instabilities have been observed in two-phase flows where the seeding density of the second phase, e.g., particles or droplets in gas, and the resulting average density, is initially non-uniform. The forcing causes the second phase to move with respect to the embedding medium. With sufficient seeding concentration, this leads to entrainment of the embedding phase. The resulting movement is similar to the movement that would evolve in a mixing flow with no second phase seeding, but with non-uniform density (not unlike a mixture of lighter and heavier gases), where RT and RM instabilities develop in the case of gravityinduced and impulsive acceleration, respectively. The hydrocode SHAMRC has been used in the past to study the formation and growth of the RM instability. Here we attempt to use it to model the first order formation and growth phenomena of the new class of instability in two-phase flows first, by approximating the second phase as a continuous fluid with an averaged density, and second, by taking the relative motion of particles (droplets) into account explicitly. The initial conditions are varied to provide a wide range of instability growth rates. Comparison of the numerical results with experiment shows good agreement.
\end{abstract}

Keywords: compressible flow, two-phase flow, instability, shock wave. 


\section{Introduction}

There exist two well-known hydrodynamic instabilities that develop in a fluid (gas) of initially non-uniform density.

The first of these two is the Rayleigh-Taylor instability (RTI), first generally described by J.W. Strutt (Lord Rayleigh) [1] for a continuous density interface, appreciably later described analytically for a sharp interface by Taylor [2], and shortly thereafter observed experimentally by Lewis [3]. Most commonly, this instability develops on a density interface between heavy and light fluid in a gravity field, when the heavy fluid is above the light fluid. The unstable (heavy above light) stratification leads to growth of perturbations on the density interface and formation of vortices, followed by development of secondary instabilities, transition to turbulence, and mixing of the heavy and light fluids. It is this interfacial instability that makes it possible, for example, to pour beer from a bottle (otherwise, the liquid would be held in by atmospheric pressure). RTI is also responsible for the formation of many natural features on Earth, such as salt domes [4] and cold plumes at subduction zones in the mantle [5]. The same instability plays an important role in stellar evolution, including such violent phenomena as Type Ia supernovæ [6].

The second instability, known as Richtmyer-Meshkov instability (RMI), also characterizes a density interface, but subjected to an impulsive acceleration, for example, due to the passage of a shock wave. It was first described analytically by Richtmyer [7]. Several years later, an experimental study of the same phenomenon by Meshkov [8] followed. RMI differs from RTI in several features. First, it develops both when the impulsive acceleration of the interface is directed from the "light" side toward the "heavy" side and in the opposite direction (in the latter case, initial perturbations will begin growing in amplitude after undergoing a phase inversion). Second, while RTI evolution is characterized by a continued supply of energy to the deforming interface (e.g., via gravity field), energy input for RMI occurs over a finite (and usually short - e.g., passage of a shock wave) time interval. Subsequently, RMI and RTI are characterized by different temporal relationships that describe perturbation amplitude growth. Like RTI, RMI is responsible for formation of some astrophysical features, e.g., in supernova remnants [9]. RMI is also important for several engineering applications, such as scramjet design [10] and inertial confinement fusion (ICF), where RMI can affect the fusion target stability [11].

Problems involving RTI and RMI are often chosen as benchmarks for validation of numerical codes [12-14] - both because they present a computational challenge and because comparison with many well-characterized experiments (or, in some cases, data describing natural phenomena) is available.

All the studies described above pertain to RTI and RMI developing on an interface (sharp or diffuse) between two liquids or gases. However, there is also a question of considerable practical (as well as theoretical) interest: what happens if, instead of a density interface between two gases, sustained or impulsive acceleration is applied to an average density interface in a multiphase medium, 
e.g., a gas non-uniformly seeded with small liquid droplets or solid particles? Modeling such a problem directly could present an even greater challenge than numerical simulation of RMI and RTI, because of the necessity to account for the interaction between the embedding (gas) and embedded (droplets or particle) phases. The simple assumption that the embedded phase can be modeled by "smearing" the mass that it adds across the appropriate volume and treating the resulting medium as a single-phase might work for some cases (especially for gravity-driven flows), but it is explicitly invalid, for example, after the passage of a shock front. The latter would accelerate the embedding phase, while particles or droplets of the embedded phase will lag behind, interacting with the gas in a possibly non-trivial way. Along with Stokes drag, compressibility effects have also to be taken into account $[15,16]$. Accounting for the interaction between each particle (droplet) and the gas could soon become computationally prohibitive.

Our recent experiments $[17,18]$ confirm formation of large-scale vortices in a gaseous medium non-uniformly seeded with micron- or submicron-sized droplets or particles after shock acceleration. The flow structure superficially resembles one that would emerge after shock acceleration of continuous medium with average initial density matching that of our two-phase, non-uniformly seeded medium. However, several important differences also exist between vortex formation due to classical RMI and the roll-up we observe.

In the following sections, we will describe two experiments - multiphase "analogues" of RTI and RMI, present some data from these experiments, and discuss results from numerical modeling of the second experiment.

\section{Experiment}

Two different experimental arrangements were built. The setup for the modeling of two-phase RTI (Fig. 1a) was extremely simple. Experiments were conducted in a tall (about $1 \mathrm{~m}$ ), fully enclosed rectangular acrylic container. In the middle of the container, horizontal grooves and a slot in the walls were machined for a removable steel bottom plate. In the top cover of the container, an opening was left for a pipe attached to a commercial theatrical fog machine, producing a stream of submicron-sized droplets of propylene glycol carried by air. Once the top section of the container was filled with droplets and the temperature of the air-droplet mix was found to be equal to the ambient (to avoid any effects due to air buoyancy), the removable bottom plate was gently extracted, allowing the seeded and unseeded air to mix. The setup was illuminated from the side with an LED panel, the flow evolution recorded with a $720 \times 480$ pixel resolution digital camera at 30 frames per second.

The arrangement for the shock-driven flow studies was by necessity much more complex (Fig. 2). At the heart of it lay a shock tube with a $75 \mathrm{~mm}$ square interior cross-section. The shock tube consisted of a $1.2 \mathrm{~m}$ long high-pressure driver section, a $2.9 \mathrm{~m}$ long driven section, a $0.8 \mathrm{~m}$ long test section (made from transparent polycarbonate), and a $0.8 \mathrm{~m}$ long runoff section. The initial conditions studied here were produced as follows. Above the test section, a 751 enclosed 

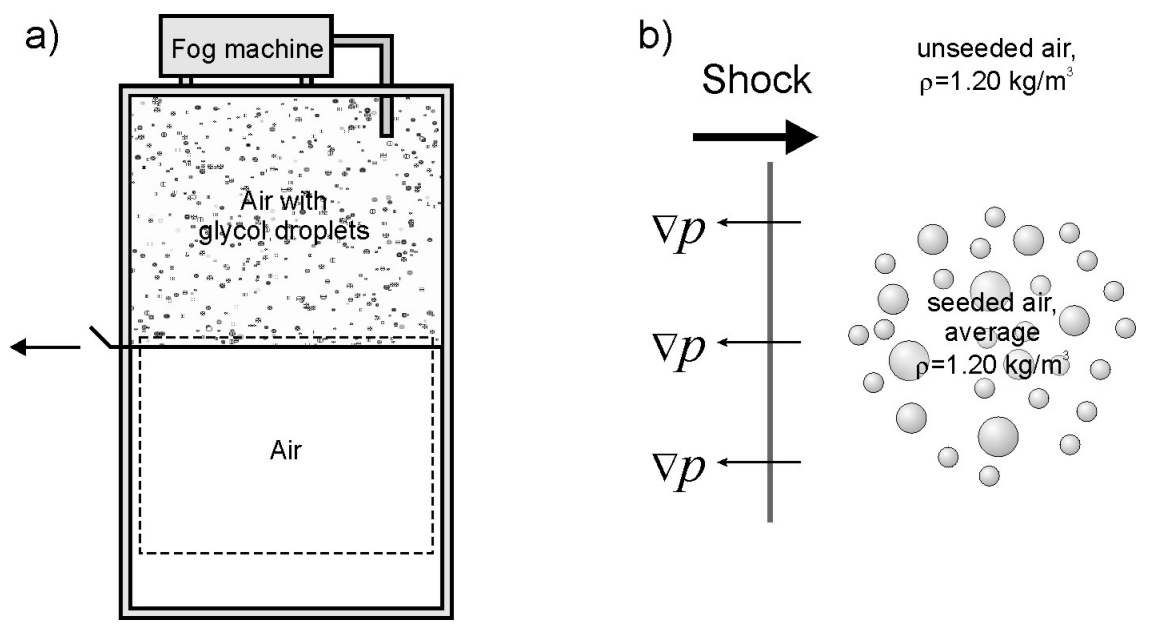

Figure 1: a) Experimental arrangement for the studies of the multiphase analogue of Rayleigh-Taylor instability. Dashed rectangle represents the field of view of the flow visualization camera. b) Schematic of initial conditions for shock-accelerated experiment.

settling tank was installed. On top of the tank, a commercial fog machine (of the same type as for the previous experiment) was mounted. The mixture of air and propylene glycol fog was allowed to reach thermal equilibrium with the surroundings before the experiments (again, to exclude air buoyancy effects). Subsequently, it was directed vertically downward across the test section through a concentric nozzle. The inner nozzle (diameter $6.35 \mathrm{~mm}$ ) contained the glycolseeded flow, while the outer concentric nozzle (outer diameter $12.7 \mathrm{~mm}$ ) carried unseeded air in the same direction. As the result, inside the test section, a laminar cylindrical jet of droplet-seeded air formed, exiting through a $12.7 \mathrm{~mm}$ hole in the bottom (Fig. 1a).

This jet formed the non-uniform droplet seeding for the initial conditions of the experiment. The shock accelerating these initial conditions was produced by pressurizing the driver section with helium and then mechanically puncturing the membrane separating the driver section from the driven section. Two pressure transducers mounted downstream in the driven section recorded pressure traces to confirm the shock speed and trigger a high-speed camera used for flow visualization (DRS Imaging IMACON-200) and the four lasers (New Wave Research Gemini) used as light sources for flow visualization. The light from each laser passed through a cylindrical lens, a spherical lens, and a flat mirror, to illuminate the same horizontal cross-section of the flow. The camera, installed on the opposite side of the test section from the lasers, was aimed at a $45^{\circ}$ mirror above the test section, recording a sequence of four frames corresponding to the four laser pulses per experiment. 

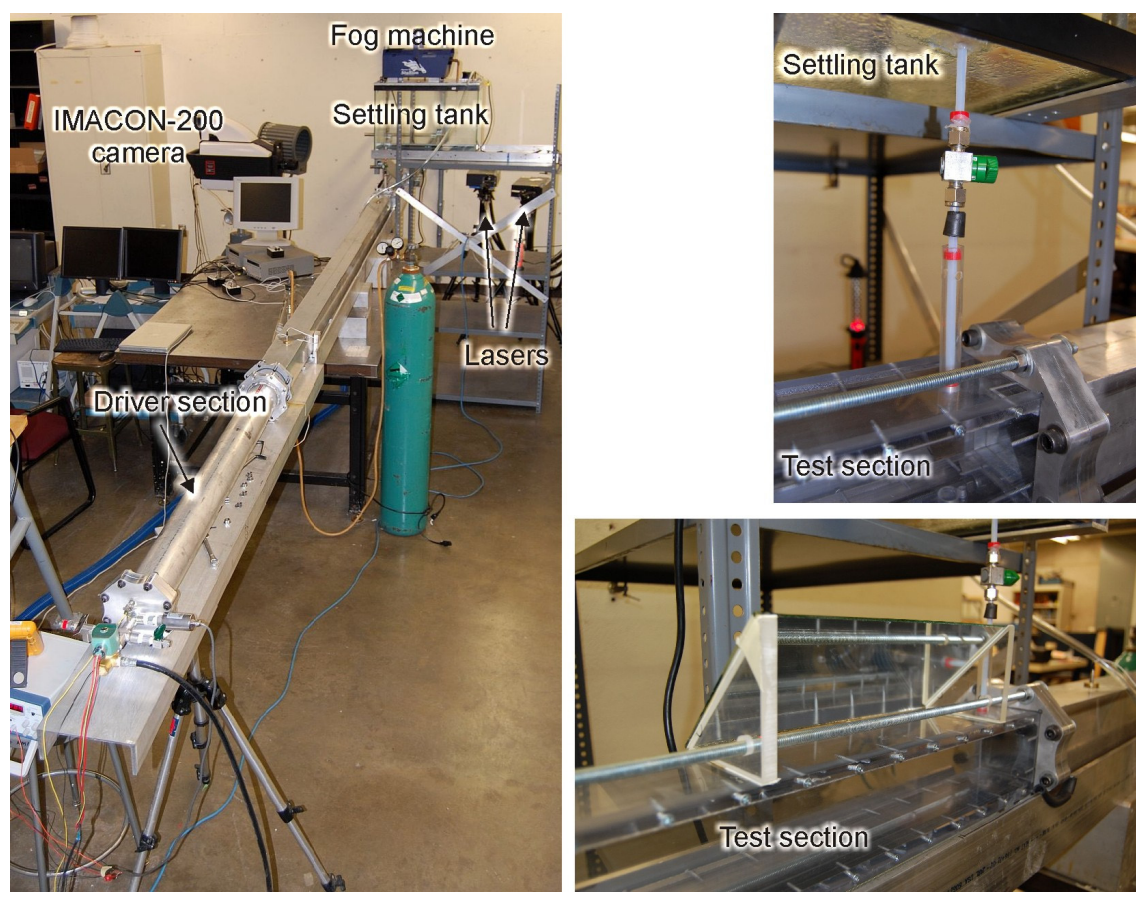

Figure 2: View of the shock tube from the upstream direction (with respect to the propagation of the shock) and close-ups of the concentric-flow nozzle and the test section with the mirror.

For the analogues of RTI and RMI described in the following section, the average initial density of the glycol-droplet mixture was measured to be $1.26 \mathrm{~kg} / \mathrm{m}^{3}$, compared to $1.20 \mathrm{~kg} / \mathrm{m}^{3}$ for the unseeded air.

\section{Numerical setup}

To expand upon knowledge gained from the RMI experiments, the Second-order Hydrodynamic Automatic Mesh Refinement Code (SHAMRC [19]) was used to model the shocks and initial conditions found in the RMI experiments. SHAMRC is a two- and three-dimensional finite-difference hydrodynamic computer code. It is a descendant of SHARC (Second-order Hydrodynamic Advanced Research Code). It is used to solve a variety of airblast related problems which include high explosive (HE) detonations, nuclear explosive (NE) detonations, structure loading, thermal effects on airblast, cloud rise, conventional munitions blast and fragmentation, shock tube phenomenology, dust and debris dispersion and atmospheric shock propagation. The code has the capability to run with a single Eulerian grid or with the Automatic Mesh Refinement (AMR) option that divides 
the calculational domain into smaller Eulerian grids at several levels of refinement to provide high-resolution results.

A two-dimensional Cartesian mesh was constructed out of square elements approximately $0.005 \mathrm{~cm}$ on each side. The size of the elements was chosen so that there would be exactly 128 zones across the diameter of the cylindrical initial conditions. The mesh extents were set to the width of the shock tube test section in the $\mathrm{y}$ direction and a distance of $51 \mathrm{~cm}$ in the $x$ direction. The $x$-extent was chosen so that approximately $2 \mathrm{~ms}$ of instability growth could be observed for shock waves with a Mach number 1.66. The shock waves were generated by a feed-in boundary condition located at the left boundary. This boundary condition provides a set of constant material properties and satisfactorily recreates the shock wave which accelerates the initial conditions.

The experimental initial conditions consisted of discrete glycol droplets suspended in ambient air. Two approaches were taken in modeling these droplets. The first was to create a pseudo-glycol fluid that has the same average mass as the droplet/air mixture. This material was modeled as a perfect gas designed to match pressure and temperature equilibrium with the surrounding ambient atmosphere. The second approach was to model droplets as discrete particles. The SHAMRC particle model treats each computational particle as a cloud of particles with the same radius. Depending of the mass of the computational particles, it may represent millions of physical particles. These particles are accelerated by the surrounding gas via drag and are allowed to heat an cool through conduction. As each computational particle has a distinct radius, a particle distribution can be generated by randomly varying the particle radius when the particles are generated. The exact distribution of the glycol droplets is an important factor, as drag is a strong function of the particle radius. Differences in particle radii will cause differences in acceleration rates and in turn cause larger particles to lag behind smaller ones. The initial droplet distribution was only approximately known (ranging from submicron-sized to several microns in diameter), so for the initial simulation a distribution was chosen with particle diameters on the order of $0.7 \mu \mathrm{m}$.

\section{Results of experiment}

Figure 3 shows a sequence of images from the video describing the two-phase RTI analogue. The most prominent features on the disturbed interface are "mushroom caps" very similar to these observed in classical RTI. From these images, the growth rate of the perturbation amplitude is well described by a quadratic function. RTI amplitude growth as the function of time $t$ is usually characterized $[20,21]$ as

$$
h(t)=\alpha A g t^{2},
$$

where $A$ is the Atwood number

$$
A=\frac{\rho_{1}-\rho_{2}}{\rho_{1}+\rho_{2}}
$$



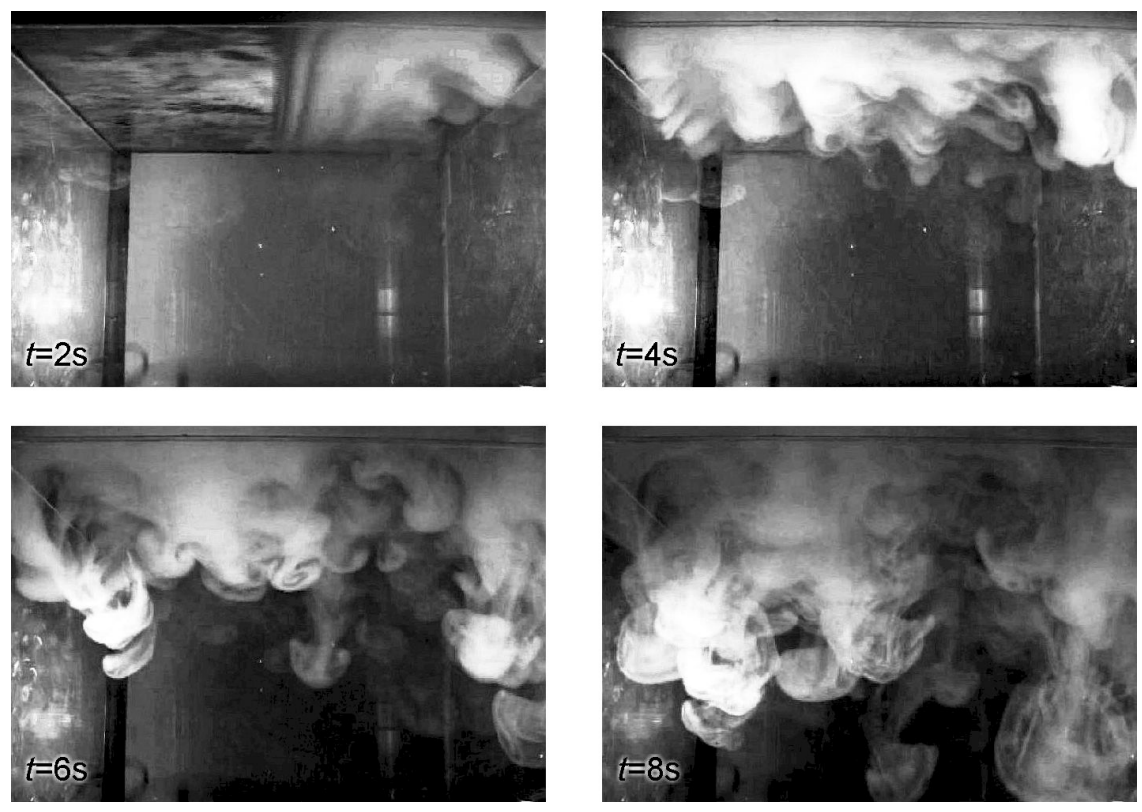

Figure 3: Flow visualization of the two-phase analogue of Rayleigh-Taylor instability. Time $t=0$ corresponds to the moment when the sliding bottom plate is set in motion. Image extent is about $50 \mathrm{~cm}$ in the horizontal direction, intervals between images $2 \mathrm{~s}$.

with $\rho_{1}$ and $\rho_{2}$ being the densities of the heavier and lighter fluid (gas) correspondingly, and $g$ is the acceleration due to gravity. In our case, $\rho_{2}$ would be the density of unseeded air, while $\rho_{1}$ would represent the average density of air seeded with droplets, leading to an effective Atwood number $A \sim 0.03$. This is a rather low value (for example, RTI with sulfur hexafluoride $\mathrm{SF}_{6}$ above air would be characterized by $A \sim 0.67$ ).

Our measurements of amplitude $h(t)$ combined with this value for $A$ yield an estimate $\alpha=0.05 \pm 0.01$, which agrees well with results for "classic" RTI [22]. The corresponding characteristic velocity can be estimated as $v=3.8 \pm 0.5 \mathrm{~cm} / \mathrm{s}$. Based on this velocity, characteristic droplet size $d=1 \mu \mathrm{m}$, and kinematic viscosity of air $\nu=1.56 \times 10^{-5} \mathrm{~m}^{2} / \mathrm{s}$, the Reynolds number $R e_{d}=d v / \nu$ for a drop is about 0.002 . At the same time, the Reynolds number of the macroscopic flow based on the representative size of the vortex structure $l \sim 2 \mathrm{~cm}$ is $R e_{l} \sim 50$. This disparity strongly suggests that the relative motion of the droplets with respect to the embedding air is negligible, and, at least from the point of view of the initial instability growth, the seeded air can indeed be regarded as continuous medium with a density $\rho_{1}$ slightly greater than that of the unseeded air $\rho_{2}$.

Thus the notion of the existence of the two-phase analogue of RTI is unambiguously confirmed. However, modeling this laboratory flow would be 

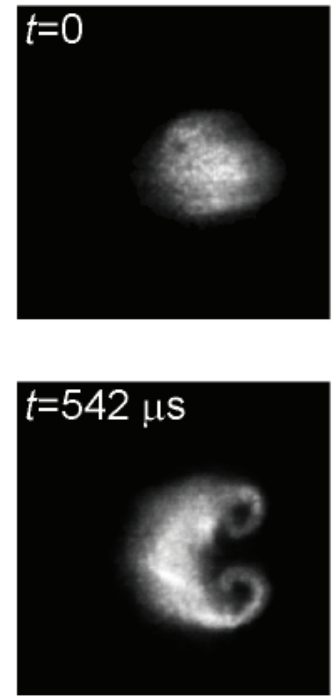
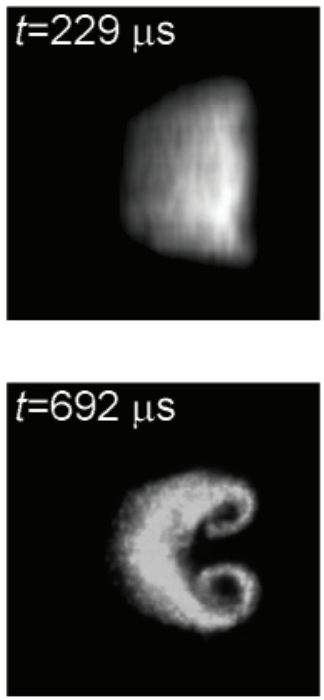
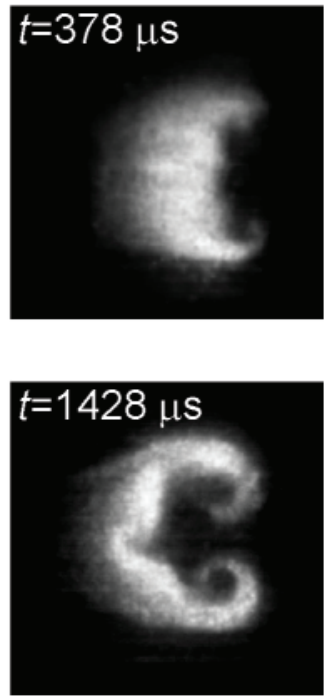

Figure 4: Experimental images acquired after Mach 1.66 shock acceleration (left to right) of a laminar, initially cylindrical air jet seeded with glycol droplets. Horizontal image extent is $20 \mathrm{~mm}$, time $t=0$ corresponds to moment immediately before shock wave arrival. Times after shock acceleration are labeled in the images. Images show a laser-illuminated horizontal section of the flow (normal to the axis of the jet). Note that the field of view follows the droplets, which are being advected by the shock-accelerated air with a piston velocity of about $300 \mathrm{~m} / \mathrm{s}$.

trivial, since continuum approach can be used. The analogue of RMI is another matter. Fig. 4 shows an image sequence acquired in several experimental runs after the shock passes through the initial conditions (droplet-seeded cylindrical jet) at Mach number $M \sim 1.66$. The bulk of the unseeded air behind the shock front in these images moves with a piston velocity of about $300 \mathrm{~m} / \mathrm{s}$. The droplets clearly do not follow the flow perfectly - they lag behind the shock-accelerated air, as the images in Fig. 4 clearly show. The "tail" of lagging droplets of larger sizes is particularly apparent in the first image following the shock passage (top row, middle, $t=229 \mu \mathrm{s}$ ). As these droplets lag behind the air, they exchange momentum with it, thereby slowing it down. This results in the air-droplet mixture reaching some equilibrium velocity that is lower than the piston velocity of air not seeded with droplets. That,in turn, leads to shear and roll-up of counter-rotating vortices.

The morphology shown evolving in Fig. 4 is both similar (counter-rotating vortex pairs) and different (droplet tail) from that evolving in the case of "classical" RMI. 


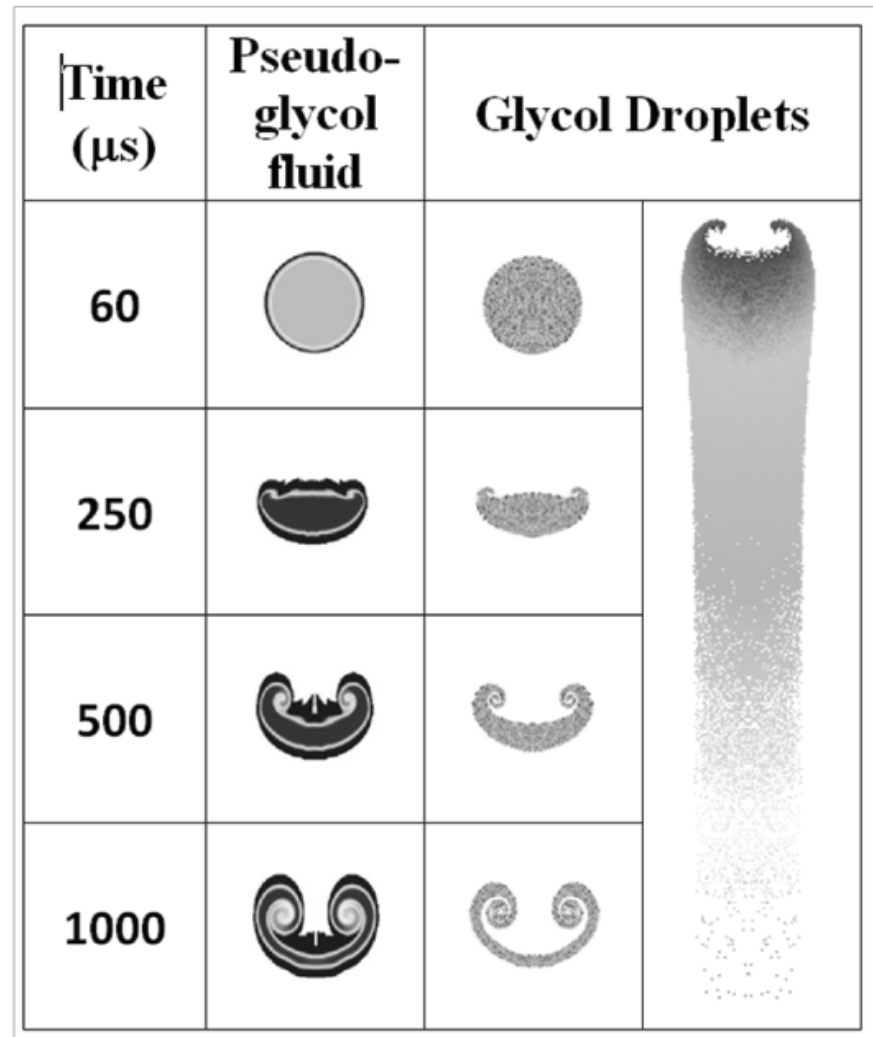

Figure 5: Numerical images of the two modeling approaches at selected times. Column 1 shows times, column 2 - results of continuum modeling, column 3 - result of modeling with computational particles of characteristic size $0.7 \mu \mathrm{m}$, column 4 - a single snapshot of the modeling result with computational particles of characteristic size $7 \mu \mathrm{m}$ corresponding to time $1000 \mu \mathrm{s}$. The shock acceleration direction is from bottom to top, horizontal cell extent is $12.7 \mathrm{~mm}$.

\section{Modeling results}

Columns two and three of Figure 5 show a series of time images from the two modeling approaches. The second column ("Pseudo-glycol fluid") represents the continuum approach, while the third and the fourth show results from modeling with particles. The difference between columns 3 and 4 is in characteristic particle size, which is increased by a factor of 10 for the last column. It is clear that both methods produce instabilities that are similar in morphology to what is observed in experiments. One aspect that is missing from the numerical results is the tail of lagging particles. This is not surprising for the pseudo-glycol fluid approach, 
but the tail should be visible in the discrete particles modeling approach. One possibility is that the particle sizes chosen for the simulation are too small. The right column of Figure 5 shows results from a run with a different particle size distribution at $1 \mathrm{~ms}$. The distribution has particle sizes an order of magnitude larger than the distribution in column three. In this new calculation, a large tail of trailing particles is formed. The tail is now much larger than that found in experiments, but this calculation demonstrates the importance of obtaining the correct particle size distribution. The roll-up of the vortices is also different in this calculation. More work is required to determine the actual particle distribution that makes up the initial conditions in the experiments and to match it with numerics.

\section{Discussion}

For "classic" RTI vs. its two-phase analogue, only in the former case is the physical gas-gas density gradient present, but this appears to be a distinction without a difference, as the embedded droplets faithfully follow the embedding air, and on the relatively short (seconds) time scale characterizing the mixing of seeded air with the unseeded air in our experiments, the flow behaves macroscopically like RTI, manifesting mixing zone growth consistent with earlier RTI experiments. The two-phase analogue of RMI is much more interesting, because after impulsive acceleration, droplets interact with the embedding phase in a complex way which presents a challenge to model accurately.

Does what we observe represent a new hydrodynamic instability? All the physical mechanisms involved are well-known, yet their combined manifestation, especially in the case of two-phase shock-accelerated flow, leads to unexpected results, whose importance may be considerable in astrophysical problems (e.g., shock propagation through dusty plasma), as well as in many engineering applications.

\section{Conclusion}

We have presented an experimental and numerical study of multiphase analogues of hydrodynamic instabilities emerging on a density gradient subject to sustained (Rayleigh-Taylor) or impulsive (Richtmyer-Meshkov) acceleration. Our work shows that vortices similarly form in a gaseous medium initially seeded with microscopic droplets so that its average density is non-uniform, and the gradient of this average density plays the role similar to the gaseous (or fluid) density gradient in classical RMI and RTI.

Future work will involve experiments with a better characterization of the initial conditions in terms of droplet sizes and distribution, as well as numerical simulations using this information. The goal of numerical exercises here is twofold. First, the code must be validated by quantitative comparison with a wellcharacterized experiment. Second, after such validation provides confidence that the numerics faithfully reproduce the relevant physics, numerical modeling can be 
applied to problems of practical interest, for example, involving propagation of a shock wave through dusty gas or plasma.

\section{Acknowledgments}

This research is funded by NNSA (US National Nuclear Security Agency) through DOE (US Department of Energy) Grant DE-PS52-08NA28920 and by DTRA (US Defense Threat Reduction Agency) awards HDTRA1-07-1-0036 and HDTRA108-1-0053.

\section{References}

[1] J.W. Strutt. Investigation of the character of the equilibrium of an incompressible heavy fluid of variable density. Proceedings of the London Mathematical Society, 14:170-177, 1883.

[2] G.I. Taylor. The instability of liquid surfaces when accelerated in a direction perpendicular to their planes. I. Proceedings of the Royal Society of London. Series A, Mathematical and Physical Sciences, 201(1065):192-196, 1950.

[3] D.J. Lewis. The instability of liquid surfaces when accelerated in a direction perpendicular to their planes. II. Proceedings of the Royal Society of London. Series A, Mathematical and Physical Sciences, 202(1068):81-96, 1950.

[4] M.P.A. Jackson and C.J. Talbot. External shapes, strain rates, and dynamics of salt structures. Geological Society of America Bulletin, 97:305-323, 1986.

[5] T.V. Gerya and D.A. Yuen. Rayleigh-Taylor instabilities from hydration and melting propel cold plumes at subduction zones. Earth and Planetary Science Letters, 212:47-62, 2003.

[6] W.H. Cabot and A.J. Cook. Reynolds number effects on RayleighTaylor instability with possible implications for type Ia supernovae. Nature Physics, 2:562-568, 2006.

[7] R.D. Richtmyer. Taylor instability in shock acceleration of compressible fluids. Communications on Pure and Applied Mathematics, 13:297-319, 1960.

[8] E.E. Meshkov. Instability of the interface of two gases accelerated by a shock wave. Izvestiya Akademii Nauk SSSR, Mekhanika Zhidkosti i Gaza, 4:151$159,1969$.

[9] A. Burrows, J. Hayes, and B.A. Fryxell. On the nature of core-collapse supernova explosions. Astrophysics Journal, 450:430-450, 1995.

[10] J. Yang, T. Kubota, and E.E. Zukoski. Applications of shock-induced mixing to supersonic combustion. AIAA Journal, 31:854-862, 1993.

[11] V.N. Goncharov. Theory of the ablative Richtmyer-Meshkov instability. Physical Review Letters, 82:2091-2094, 1999.

[12] R.M. Baltrusaitis, M.L. Gittings, R.P. Weaver, R.F. Benjamin, and J.M. Budzinski. Simulation of shock-generated instabilities. Physics of Fluids, 8:2471-2483, 1996. 
[13] A.W. Cook and Y. Zhou. Energy transfer in Rayleigh-Taylor instability. Physical Review E, 66:026312-1 026312-12, 2002.

[14] A. Palekar, P. Vorobieff, and C.R. Truman. Two-dimensional simulation of Richtmyer-Meshkov instability. Progress in Computational Fluid Dynamics, 7(8):427 - 438, 2007.

[15] M.K. Parmar, A. Haselbacher, and S. Balachandar. Prediction and modeling of shock-particle interaction. AIAA Paper 2009-1124, 2009.

[16] M.K. Parmar, A. Haselbacher, and S. Balachandar. Improved drag correlation for spheres and application to shock-tube experiments. AIAA Journal, 48:1273-1276, 2010.

[17] M. Anderson, P. Vorobieff, S. Kumar, J. Conroy, R. White, C. Needham, and C.R. Truman. Numerical simulation of a shock-accelerated multiphase fluid interface. To be published in Proceedings of 28th International Symposium on Shock Waves, 2011.

[18] P. Vorobieff, M. Anderson, J. Conroy, R. White, C.R. Truman, and S. Kumar. Vortex formation in shock-accelerated gas induced by particle seeding. Submitted to Physical Review Letters, 2011.

[19] J.E. Crepeau. SHAMRC, second-order hydrodynamic automatic mesh refinement code, vol. 1: Methodology. Applied Research Associates, Albuquerque, NM, USA, 1999.

[20] G. Birkhoff. Taylor instability and laminar mixing. University of California Report No. LA-1862, 1955.

[21] D.L. Youngs. Numerical simulation of turbulent mixing by Rayleigh-Taylor instability. Physica D: Nonlinear Phenomena, 12:32-44, 1984.

[22] P. Ramaprabhu, G. Dimonte, and M.J. Andrews. A numerical study of the influence of initial perturbations on the turbulent RayleighTaylor instability. Journal of Fluid Mechanics, 536:285-319, 2005. 